\title{
Approaches to outline the aromatic profile of Kyoho wines from South Korea
}

\author{
Enoch Yang, Foteini Drosou, Euthalia G. Dourtoglou, Archodoula Chatzilazarou, and Vassilis G. Dourtoglou ${ }^{\mathrm{a}}$ \\ Department of Oenology and Beverage Technology, Faculty of Food Technology and Nutrition, Technological Educational Institute of \\ Athens, 12 Ag. Spyridonos St., Egaleo, 12210 Athens, Greece
}

\begin{abstract}
Kyoho cultivar is a hybrid of $V$. vinifera and $V$. labrusca and is one of the major varieties cultivate in South Korea. Kyoho grapes are characterized for their intense grape flavor and sour attributes. Korean climatic conditions are different from worldwide wine producing countries. The summer season is hot and dominated by heavy rains. Wines from Kyoho grapes are distinct for their intense grape flavor and the key odors that characterize the wines are aromas of caramel and strawberry notes. The past few years efforts are put to improve the quality and to advance the potential of the domestic wines. The objective of the present study is to provide insight to the main aromatic compounds that form the aroma profile of Kyoho wines. For this aim, Gas Chromatography was coupled with Mass Spectrometry for the separation and identification of the compounds. Analysis allowed to identify the volatile compounds that form the aroma of the wine and to detect the key odors of its characteristic aroma. (2,5-dimethyl-4-hydroxy-3(2H)-furanone) Furaneol ${ }^{\circledR}$, which is associated with caramel-like flavor, was one of the compounds that were detected along with ((3R)-hydroxy4,4-dimethyloxolan-2-one) pantolactone.
\end{abstract}

\section{Introduction}

The past years the grape industry in Korea has been showing a positive trend in terms of increase area and production volume. Korean grapes are mainly produced for table use and only a small portion is used for wine production. Owing to hot and rainy weathers in the summer and cold conditions in winter, the climatic conditions in Korea are unsuitable for the growth of $V$.vinifera. [1] Thus, Korean wine makers try using table grape from $V$. labrusca cultivars and their hybrids to produce wines, as American grapes show stronger adaptation to the adverse weather conditions [1,2].

Main grapes growing in Korea are, 'Campbell Early' which occupies $74.3 \%$ of the total production area, 'Kyoho' which occupies $13.1 \%$ and 'Muscat Bailey A' which covers a small portion of $5.9 \%$ of the total area cultivated for grape production [1,3]. The majority of Korean domestic wines are produced using the most cultivated variety, that is Campbell Early. Though it has the limitations of containing low levels of sugar, high malic acid concentration and significant foxy aroma content that affects negatively its preference [4]. Kyoho, the second most cultivated grape, is a tetraploid hybrid of $V$. labrusca and $V$. vinifera ('Ishihara Wase' $\times$ 'Centennial') [5-7] and it is famous for its large-sized berry and for its intense grape flavor [5]. Several studies have been conducted for the improvement and optimization of the wines produced from V. labrusca grapes. Hong \& Park [4] analyzed the diversity of indigenous yeasts isolated from Campbell Early grapes. Furthermore, the identified yeast strains were examined for their tolerance to potassium metabisulfite

a e-mail: vdourt@teiath.gr as well as their alcohol and flavor production [4]. Ahn et al. [8], examined the physiochemical differences between V.vinifera and V. labrusca grapes by analyzing their organic acid contents. Son et al. [9] used targeted analysis of ${ }^{1} \mathrm{H}$ NMR to characterize and differentiate between $V$. labrusca grapes and their wines. In their study, Kyoho wines presented the highest levels of glycerol, 2,3-butanediol, succinate and alcohol compared to other V. labrusca wines. Lee et al. [10] evaluated the sensory attributes of a mixture of two different grape varieties, Kyoho and Campbell Early and its chemical attributes were determined and compared to a French Beaujolais wine. Principal component analysis of the descriptive data, showed high intensities of "ripe-fruit aroma", "caramel aroma" and "sour attributes for Kyoho wines.

In this study, the volatile compounds of Kyoho wines were analyzed to determine which groups of compounds consist the aroma profile of the wines and which compounds contribute to the grape/caramel-like odors of the wines.

\section{Material and methods}

\subsection{Chemicals}

Dichloromethane, chloroform, sodium chloride and anhydrous sodium sulphate were purchased from Chem Lab (Zedelgem, Belgium). (2,5-dimethyl-4-hydroxy-3(2H)furanone) Furaneol ${ }^{\circledR}$ was purchased from Sigma-Aldrich (Athens, Greece).

\subsection{Samples}

For the fulfillment of this study four monovarietal Kyoho wines of two different produces were examined (ES and 
GS). From each producer two wine samples were provided (ES1, ES2 and GS1, GS2, respectively). The grapes were obtained and produced in Cheonan, Korea.

\subsection{Volatile analysis}

The extraction of volatile compounds was carried away by Liquid-Liquid extraction (LLE) using dichloromethane as elution sorbent. For the analysis of the volatile compounds of the each examined wine, $50 \mathrm{~mL}$ of sample, $10 \mathrm{~mL}$ of saturated $\mathrm{NaCl}$ solution and $50 \mathrm{~mL}$ of dichloromethane were added in laboratory flask. The flask was stirred for $10 \mathrm{~min}$ at room temperature. Then, the sample was centrifuged at $3500 \mathrm{rpm}$ for $5 \mathrm{~min}$ for the separation of phases. The supernatant was then extracted again using the same volume of solvent for $10 \mathrm{~min}$. The organic layers were then washed with distilled water in a separation funnel. The organic phase was dried over anhydrous sodium sulphate, filtered and then condensed in a vacuum rotary evaporator. Prior to instrumental analysis, the sample was recovered to $50 \mu \mathrm{L}$ with Chloroform. For the injection $1 \mu \mathrm{L}$ was used. Each sample was analyzed in duplicate.

The identification of examined compounds was made using their retention time and by comparing their mass spectra with a spectral library of known standard compounds. For the quantification of (2,5-dimethyl-4-hydroxy-3(2H)-furanone) Furaneol ${ }^{\circledR}$ external calibration curves were prepared from standards. For the quantification of ((3R)-hydroxy-4,4-dimethyloxolan2-one) Pantolactone and (2,5-dimethyl-4-methoxy-3(2H)furanone) Mesifuran, the area of the corresponding peaks was normalized by that of the corresponding internal standard. The quantification of the compounds was made in Full Scan. The internal standard used for quantification was 3-Octanol $(3.0 \mathrm{mg} / \mathrm{L})$. The $\mathrm{GC}$ peak area of each compound was obtained from the ion extraction chromatogram by selecting target ions for each one.

\subsection{Gas Chromatography/ mass spectrometry analysis}

The apparatus used was an Agilent 6890 series GC System (Agilent Technologies, USA), equipped with 5975C VL MSD and a fused silica capillary column, $30 \mathrm{~m} \times$ $0.32 \mathrm{~mm}$ i.d. $\times 0.25 \mu \mathrm{m}$ film thickness (HP-5MS, Agilent Technologies). $1.0 \mu \mathrm{L}$ of sample was injected using a split ratio of 100:1. The injector temperature was set at $250^{\circ} \mathrm{C}$, the carrier gas was helium at a flow rate of $1 \mathrm{~mL} / \mathrm{min}$, and the oven temperature program was $50^{\circ} \mathrm{C}$ for $2.5 \mathrm{~min}$, increased to $180^{\circ} \mathrm{C}$ at $2.5^{\circ} \mathrm{C} / \mathrm{min}$, then to $230^{\circ} \mathrm{C}$ at $2^{\circ} \mathrm{C}$ $/ \mathrm{min}$, then to $250^{\circ} \mathrm{C}$ at $6^{\circ} \mathrm{C} / \mathrm{min}$, then the temperature was held at $250^{\circ} \mathrm{C}$ for 5 minutes, then increased to $270^{\circ} \mathrm{C}$ at $5^{\circ} \mathrm{C} / \mathrm{min}$ and lastly it was held for 2 mins at $270^{\circ} \mathrm{C}$. The temperature of the transfer line was set at $280^{\circ} \mathrm{C}$. The mass spectrometer operated in the ionization mode (EI) at an ionization voltage of $70 \mathrm{eV}$ in the mass range $40-550 \mathrm{amu}$ and a manifold temperature of $270^{\circ} \mathrm{C}$. All data was recorded using the Turbomass 5.0 ChemStation software.

\section{Results and discussion}

\subsection{Aroma volatiles composition by GC-MS}

A total of 52 compounds were identified across four Kyoho wine samples. The determined compounds are summarized and classified by their functional groups in Table 1. Among the determined compounds, the group with the most compounds identified were higher alcohols and esters. Altogether 16 alcohols, 15 esters, 7 fatty acids, 3 furans, 2 lactones and 3 miscellaneous compounds were detected.

Higher alcohols, also known as fusel alcohols, are, from a quantitative point of view, the most important group of volatile compounds produced by yeast during alcoholic fermentation. [11] Among the higher alcohols in wines, compounds which exhibit the greatest abundance are isoamyl alcohol 2-phenylethanols. 2-phenylethanol is one of the most important phenol-derived higher alcohol and it produces rose-like fragrances. [12-14]. Likewise, Kyoho wines presented significant peaks for the abovementioned compounds.

Esters are mainly by-products of the alcoholic fermentation and they are considered important to the sensory properties of wines, as they contribute fruity notes. Among the detected esters in the examined wines, major compounds which are known to contribute to the fruity character of a wine are isoamyl acetate, 2-phenyl acetate, ethy hexanoate and ethyl decanoate. $[13,15]$ These compounds were determined in all samples. Iso-butyl acetate which has a strawberry-like odor was detected only in one wine as shown in Table 1.

Fatty acids are mainly formed by yeasts during alcoholic fermentation. Volatile fatty acids tend to have a negative effect on wine aroma, contributing green and cheese, fatty aromas $[12,13]$. Some of the fatty acids that were identified in the examined wines were octanoic and decanoic acids. Free fatty acids occur only as traces in grapes, but they are biosynthesized during fermentation by yeasts and bacteria. Considering the compounds' unpleasant odors and similar ranges of concentrations across wines, these acids are not considered to be important contributors to the flavor of these wines [10].

Among phenolics, Eugenol was detected which is characterized as clove-like note. At usual concentrations, eugenol adds a general spic note as well [16].

In the examined Kyoho wines two compounds containing the 1-oxacycloalkan-2-one structure were identified; $\gamma$-butyrolactone and pantolactone. Concentrations of pantolactone were from 0.063 to $0.145 \mathrm{mg} / \mathrm{L}$. Pantolactones appear to be generated in grapes under carbonic maceration, derived from amino or organic acids, notably glutamic and succinic acids [14]. $\gamma$-Butyrolactone, has been suggested that it contributes to the aroma characteristics of Sherry wines, giving pleasant cotton, peachy-like odors [11].

Contrast to major substances, minor classes can contribute greatly to the flavor of the wine differentiating each wine. Such classes are furans. Furans have very sweet aromas and are normally classified in the same group of aromas with give an odor of "burnt" sugar and caramel. [17] Furaneol ${ }^{\circledR}$, pantolactone and (2,5-dimethyl4-methoxy-3(2H)-furanone) mesifuran, compounds that contribute to a sweet odor, were detected in all wines, except for mesifuran which was not found in GS2. The quantified concentrations of all three compounds are listed in Table 2.

The quantities of mesifuran determined in the Kyoho wines ranged between 0.046 to $0.066 \mathrm{mg} / \mathrm{L}$. It is reported 
Table 1. Volatile compounds detected in Kyoho wines.

\begin{tabular}{|c|c|c|c|c|c|}
\hline Compound & ES1 & ES2 & GS1 & GS2 & Odor description \\
\hline \multicolumn{6}{|l|}{ Higher alcohols } \\
\hline 1-Butanol & - & - & - & + & Intoxicant alcohol $^{\mathrm{b}}$ \\
\hline Isobutanol & - & - & - & + & Fusel, alcohol ${ }^{\mathrm{b}}$ \\
\hline 1-Pentanol & - & - & + & + & Fusel alcohol, sweet balsam ${ }^{c}$ \\
\hline Iso-Amyl alcohol & + & + & + & + & Harsh, fruity, banana ${ }^{a, c}$ \\
\hline Prenol & + & + & + & + & Fruity, green lavender ${ }^{\mathrm{c}}$ \\
\hline 3-methyl-1-pentanol & - & + & + & + & Alcohol, fusel, cognac, green fruity ${ }^{\mathrm{b}, \mathrm{c}}$ \\
\hline Isohexanol & - & + & + & + & Nutty $^{\mathrm{c}}$ \\
\hline 1-Hexanol & + & + & + & + & Green grass ${ }^{\mathrm{a}}$ \\
\hline 3-Hexen-1-ol & + & + & + & + & Green leafy ${ }^{c}$ \\
\hline 2-Ethyl-1-hexanol & + & + & + & + & Mushroom, citrus floral ${ }^{\mathrm{b}, \mathrm{c}}$ \\
\hline 1-Heptanol & - & - & - & + & Fragrant plant ${ }^{\mathrm{b}}$ \\
\hline Benzyl alcohol & + & + & + & + & Floral, rose $\mathrm{e}^{\mathrm{c}}$ \\
\hline Phenylethyl alcohol & + & + & + & + & Floral, rose, rose water ${ }^{\mathrm{a}, \mathrm{c}}$ \\
\hline Tyrosol & + & + & + & + & Mild sweet, floral, fruity ${ }^{c}$ \\
\hline Homovanillyl alcohol & + & + & + & + & N.f. \\
\hline Tryptophol & + & + & + & + & N.f. \\
\hline \multicolumn{6}{|l|}{ Esters } \\
\hline Ethyl Lactate & + & + & + & + & Solvent ${ }^{\mathrm{a}}$ \\
\hline Iso-Butyl acetate & - & - & - & + & Strawberry, fruity, flowery ${ }^{\mathrm{b}}$ \\
\hline Ethyl Butanoate & + & + & + & + & Sour fruit, strawberry ${ }^{\mathrm{b}}$ \\
\hline Isoamyl Acetate & + & + & + & + & fresh banana \\
\hline Isoamyl Lactate & + & + & + & + & Sharp tart, buttery, butterscotch ${ }^{\mathrm{c}}$ \\
\hline Ethyl Hexanoate & + & + & + & + & Green apple, fruity ${ }^{\mathrm{b}}$ \\
\hline Diethyl Succinate & + & + & + & + & Light fruity ${ }^{\mathrm{b}}$ \\
\hline Methyl vanillate & + & + & + & + & Warm spicy, vanilla ${ }^{\mathrm{c}}$ \\
\hline Ethyl vanillate & + & + & + & + & Phenolic, burnt, smoky ${ }^{\mathrm{c}}$ \\
\hline Ethyl octanoate & + & + & + & + & Melon, pineapple $\mathrm{a}^{\mathrm{a}, \mathrm{b}}$ \\
\hline Phenethyl acetate & + & + & + & + & Honey, pollen, rose ${ }^{\mathrm{b}}$ \\
\hline Ethyl Decanoate & + & + & + & + & Fruity, fatty, pleasant ${ }^{\mathrm{b}}$ \\
\hline Succinic acid, ethyl 3-methylbutyl ester & + & + & + & + & N.f. \\
\hline Ethyl Dodecanoate & + & + & - & - & Fruity, fatty, pleasant ${ }^{\mathrm{b}}$ \\
\hline Ethyl palmiate & + & + & + & + & Waxy, fruity, creamy ${ }^{\mathrm{c}}$ \\
\hline \multicolumn{6}{|l|}{ Acids } \\
\hline Octanoic acid & + & + & + & + & Rancid, harsh, cheese ${ }^{c}$ \\
\hline Decanoic acid & + & - & + & + & Fatty, unpleasant ${ }^{\mathrm{c}}$ \\
\hline Tetradecanoic acid & + & + & + & + & N.f. \\
\hline Hexadecanoic acid & + & + & + & + & N.f. \\
\hline Octadecanoic acid & - & + & - & - & N.f. \\
\hline Homovanillic acid & + & + & + & + & N.f. \\
\hline Homo-syringic acid & + & + & + & + & N.f. \\
\hline \multicolumn{6}{|l|}{ Phenols } \\
\hline Phenol, 2,5-di-tert-butyl- & + & + & + & + & N.f. \\
\hline Eugenol & + & + & + & + & Clove $^{\mathrm{c}}$ \\
\hline Phenol, 2,6-dimethoxy-4-(2-propenyl) & + & + & - & - & N.f. \\
\hline 4-((1E)-3-Hydroxy-1-propenyl)-2-methoxyphenol & + & + & + & + & N.f. \\
\hline Phenol, 2,4-bis(1,1-dimethylethyl)- & + & + & + & + & N.f. \\
\hline \multicolumn{6}{|l|}{ Furans } \\
\hline 2-Furanmethanol & - & - & + & + & Piquancy, bitter ${ }^{\mathrm{b}}$ \\
\hline Furaneol ${ }^{\circledR}$ & + & + & + & + & Caramel $^{\mathrm{b}, \mathrm{c}}$ \\
\hline Mesifuran & + & + & + & - & Sweet, maple, fruity ${ }^{\mathrm{c}}$ \\
\hline \multicolumn{6}{|l|}{ Lactones } \\
\hline Pantolactone & + & + & + & + & Cotton candy ${ }^{c}$ \\
\hline$\gamma$-Butyrolactone & + & + & + & + & Creamy, peach ${ }^{\mathrm{d}}$ \\
\hline \multicolumn{6}{|l|}{ Miscellaneous } \\
\hline 6R,9R-3-Oxo- $\alpha$-ionol & + & + & - & + & Spicy $^{\mathrm{c}}$ \\
\hline Limonene & - & - & + & + & Lemon, orange $^{a}$ \\
\hline 3-Hydroxy-beta-damascone & + & + & - & + & N.f. \\
\hline
\end{tabular}

N.f.: not found

${ }^{a}$ Refers to Wang et al. (2016) [13]

${ }^{\mathrm{b}}$ Refers to Wang et al. (2017) [19]

${ }^{\mathrm{c}}$ Obtained from the Good Scents Company, http://www . thegoodscentscompany . com [20]

${ }^{\mathrm{d}}$ Refers to Pereira et al. (2014) [21]. 
Table 2. Concentrations of quantified volatile compounds $(\mathrm{mg} / \mathrm{L})^{a}$.

\begin{tabular}{lcccc}
\hline & ES1 & ES2 & GS1 & GS2 \\
\hline Furaneol $^{\circledR}$ & 0.384 & 0.459 & 0.066 & 0.098 \\
Mesifuran & 0.046 & 0.063 & 0.066 & N.d. \\
Pantolactone & 0.098 & 0.094 & 0.063 & 0.145 \\
\hline
\end{tabular}

N.d.: Not detected

${ }^{a}$ Means are derived from duplicate determinations.

that mesifuran has been detected in substantial amounts in V. labrusca hybrids often giving a caramel-like undertone [18].

The determined concentration of Furaneol ${ }^{\circledR}$ ranged between 0.066 to $0.459 \mathrm{mg} / \mathrm{L}$. As stated by Rapp et al. (1980) in [17], Furaneol ${ }^{\circledR}$ can be found in quantities above $1 \mathrm{mg} / \mathrm{L}$ in wines elaborated with hybrid grapes; concentrations at which it produces a disagreeable strawberry scent. In our case, although the examined wines of our study did not present concentrations above $1 \mathrm{mg} / \mathrm{L}$, the wines had a predominant odor of strawberries and caramel-like notes, which can be imparted to the presence of Fureaneol and Mesifuran. Similar sensory attributes were described in the work of Lee et al. [10]. A blend of Kyoho and Campbell Early (V. labrusca varieties) wines were evaluated organoleptically; descriptive analysis showed high correlation for grapiness, sweetness and sourness. Likewise, Jackson [16] mentions that it is widely referred for V. labrusca grape cultivars to possess intense strawberry odor, which is probably induced by furaneol ${ }^{\circledR}$ and tis methoxy derivatives.

Other wines in which Furaneol ${ }^{\circledR}$ imparts a significant role are rose wines from Provence. Masson \& Schneider, [15] identified that the key compounds contributing to the distinct aroma of Provence rose wines are derived by the furanic compounds furaneol ${ }^{\circledR}$ and homofuraneol, the $\mathrm{C} 13$ norisoprenoid ( $\beta$-damascenone) and the two volatile thiols 3-mercaptohexanol and 3-mercaptohexyl acetate. In their study, the wines examined presented high concentrations of furaneol ${ }^{\circledR}$ which indicate that furaneol ${ }^{\circledR}$ play a significant role in the aroma of rose wines. Similarly, other authors in [13] affirm that the key compounds of rose wines are ethyl esters, higher alcohol acetates (particularly isoamyl acetate and 2-phenyl acetate), furaneol ${ }^{\circledR}$ and the polyfunctional thiols 3-mercapto-1-hexanol and 3-mercaptohexyl acetate. In our study polyfunctional thiols were not detected but it could be due to inadequate sensitivity in the extraction procedure as the polyfunctional thiols are present in extremely low concentrations [13]. Besides these compounds, $\beta$-damascenone is considered to contribute significantly in the rose wine key odors enhancing their fruity notes [15]. Taking into consideration the fact that Kyoho grapes do not produce deep red wines, but are weak in color as stated in [10], attempts to produce rose wines could be encouraged, since they present similar key odors to the Provence rose wines.

\section{Conclusions}

In summary, this study aimed to determine the volatile compounds of Kyoho grapes. Specifically, this work involved comprehensive evaluation of 4 Kyoho wines, in which its sweet, strawberry, caramel like odor were identified; these sweet aromas are characteristic of all $V$. labrusca grapes even in their hybrid grapes showing intense grape flavor, roses and strawberry like odors [14], which are imparted by furans like furaneol ${ }^{\circledR}$ and its methoxy derivatives.

This research has been co-funded by the European Union (European Social Fund-ESF) and Greek national funds through the Operational Program "Education and Lifelong Learning" of the National Strategic Reference Framework (NSRF) - Research Funding Program: ARCHIMEDES III. Investing in knowledge society through the European Social Fund.

\section{References}

[1] H. K. Yun \& K. S. Park, Int. J. Pl. Brd., 1(1), 22-26 (2007)

[2] M. B. M. de Castilhos, O. L. dos Santos Correa, M. C. Zanus, J. D. G. Maia, S. Gomez-Alonso, E. Garcia-Romero, V. L. Del Bianchi \& I. Hermosin-Gutierrez., Food Res. Intr. 76(3), 697-708 (2015)

[3] H.J. Ahn \& H.S. Son, Korean J. Food Sci. Tech. 44(3), 280-286 (2012)

[4] Y. Hong \& H. Park, Foo. Micr. 34, 297-214 (2013)

[5] Y. Deng, Y. Wu \& Y. Li, LWT 38, 584-590 (2006)

[6] S. Shiraishi, Y. Watanabe, H. Okubo \& S. Uemoto, J. Japan. Soc. Hort. Sci. 55(2), 123-129 (1986)

[7] M. Shiraishi, R. Shinomiya \& H. Chijiwa, Sci.Hort. 137, 107-113 (2012)

[8] H. Ahn \& H. Son, Kr. J. Food Sci. Tech. 44(3), 280$286(2012)$

[9] H. Son, G. Hwang, H. Ahn, W. Park, C. Lee \& Y. Hong, Food Res. Intr. 42, 1483-1491 (2009)

[10] S. Lee, J. Lee, H. Kim, S. Kim \& K. Koh, Food Chem. 94, 385-393 (2006)

[11] M. V. Moreno-Arribas \& M. Carmen Polo, Wine Chemistry and Biochemistry (2009)

[12] F. Vararu, J. Moreno-Garcia, C. Zamfir, V. V. Cotea \& J. Moreno, Food Chem. 197, 373-381 (2016)

[13] J. Wang, D. L. Capone, K. L. Wilkinson \& D. W. Jeffery, Food Chem. 196, 682-693 (2016)

[14] R. S. Jackson, Wine Science: Principles and Applications (2008)

[15] G. Masson \& R. Schneider, A.J.E.V. 60(1), 116-122 (2009)

[16] R. S. Jackson, Wine Tasting A Professional Handbook (2009)

[17] V. Ferreira, I. Jarauta, R. Lopez \& J. Cacho,J. Chrom. A 1010, 95-103 (2003)

[18] I. Zabetakis, J. W. Gramshaw \& D. S. Robinson, Food Chem. 65(2), 139-151 (1999)

[19] X. Wang, Y. Tao, Y. Wu, R. An \& Z. Yue, Food Chem. 226, 41-59 (2017)

[20] http: //www . thegoodscentscompany.com/

[21] V. Pereira, J. Cacho \& J. C. Marques, Food Chem. 162, 122-134 (2014) 